\title{
Adaptive Control for Reversing a Two-Vehicle Platoon
}

\author{
Plamen Petrov Michel Parent \\ INRIA/IMARA - Domaine de Voluceau-Rocquencourt-BP 105 \\ 78153 Le Chesnay cedex, France \\ E-mail: pp_petrov@yahoo.com_Michel.Parent@inria.fr
}

\begin{abstract}
This paper presents an adaptive tracking controller for a two-vehicle convoy where the lead vehicle is driven in reverse. We assume that the lead vehicle linear and angular velocities are unknown constant parameters. We consider the problem of autonomous vehicle tracking without the use of road infrastructure or inter-vehicle communication. The only information the robot vehicle can use for feedback control is the relative position and orientation with respect to the lead vehicle obtained from onboard sensing. The control velocities of the ego-vehicle are computed using the leader velocity estimates obtained from the dynamic part of the proposed controller. The proposed adaptive control law achieves asymptotic stabilization of the closed-loop system in error coordinates. For constant velocity maneuvers of the leader, at steady state, the two-vehicle convoy will travel concentric arcs of same radii with prescribed inter-vehicle spacing. Simulation results are presented to illustrate the effectiveness of the proposed controller.
\end{abstract}

Keywords: Autonomous vehicles; Backtracking; Adaptive Control

\section{INTRODUCTION}

In recent years, there has been considerable interest in controlling robotic vehicles in a convoy-like fashion, where each vehicle tracks the path taken by the preceding car at a desired separation distance. This scenario is very useful in the case of military convoys (Cheok, et al. 1997; Brumitt, et al. 1998; Hogg, et al. 2001), convoys of commercial vehicles (Fritz, 1999) or in the public urban transport (Parent and Daviet, 1996). While the problem of automatic vehicle following in the case of forward driving has attracted a great deal of attention during the last decade (Lu, et al., 2004; White et al., 2001; Canudas-de-Wit and Doudi-Likho, 2000), to our knowledge, the problem of backward driving has seldom been addressed in the literature (Artus, et al. 2003). The importance of integrating the ability of backward driving in the leader-tracking scenario arises from many practical situations where the vehicle convoy operates in structured or natural terrains. For example, when the lead vehicle is too far inside a cul- de-sac, reverse motion is unavoidable in order to prevent the failure of the mission.

This paper presents an adaptive tracking controller for two-vehicle convoy where the lead vehicle is driven in reverse. We assume that the lead vehicle linear and angular velocities are unknown constant parameters. The leader is under human or automatic control. We consider the problem of autonomous vehicle tracking without the use of road infrastructure or inter-vehicle communication. The only information the robot vehicle can use for feedback control is the relative position and orientation with respect to the lead vehicle obtained from onboard sensors (either vision-based or radarbased) which monitor the rear end of the lead vehicle.

One of the challenges in designing controllers for automatic vehicle tracking where the lead vehicle is driven in reverse is to decide the desired trajectory of the ego-vehicle. In order to maintain a desired intervehicle spacing, a possible scenario consists in tracking a reference virtual frame behind the lead vehicle, which is rigidly positioned on its longitudinal axle. However, 
on a curved section of constant radius (all curves are assumed to have constant radius), at steady state, the ego-vehicle will travel an arc concentric to that traveled by the lead car but with bigger radius and this may be unacceptable from a practical point of view.

Assuming constant velocity maneuvers of the leader, in this paper, we are interested in designing a nonlinear adaptive controller for the robot vehicle, such that, at steady state, the two-vehicle convoy will travel concentric arcs of same radii with prescribed (desired) inter-vehicle spacing depending on linear and angular velocities of the leader. A speed-dependent inter-vehicle spacing policy is introduced. The desired spacing is a function of the leader linear and angular velocity estimates and an a priori known desired inter-vehicle spacing which is defined for the case of straight line maneuver.

We consider the relative leader/follower dynamics in leader coordinate frame where the system outputs are the position and the orientation of the robot vehicle with respect to the leader (measured by onboard sensing). Using the leader velocity estimates obtained from the dynamic part of the proposed controller, we formulate a reference output for the system. The dynamic part of the controller is designed as a parameter update law. The control objective is to asymptotically track the reference output with the system output in the presence of unknown constant values of the leader linear and angular velocities. The proposed adaptive control law designed on a reduced order dynamics combined with high-gain design method, achieves asymptotic stabilization of the closed-loop system in error coordinates. The control velocities of the ego-vehicle are computed using the leader velocity estimates. For constant velocity maneuvers of the leader, at steady state, the two-vehicle convoy will travel concentric arcs of same radii with prescribed inter-vehicle spacing. Throughout this paper, feedback control design and stability analysis are performed via Lyapunov techniques.

The rest of the paper is organized as follows. In Section 2, the control model based on the relative vehicle dynamics is presented and the control objective is formulated. In Section 3, we present the adaptive tracking controller for backward driving of a twovehicle convoy. Section 4, contains simulation results which illustrate the effectiveness of the proposed controller. Conclusions are presented in Section 5.

\section{PROBLEM FORMULATION}

\subsection{Relative Kinematics}

In order to design the control law, we need to know the equations governing the motion of the egovehicle in the frame of the lead vehicle. In this section, we derive the relative kinematics between the leader (called also "first vehicle", in this paper) and the egovehicle (second vehicle). We consider a two-vehicle convoy moving on a horizontal plan, as shown in Fig.1.

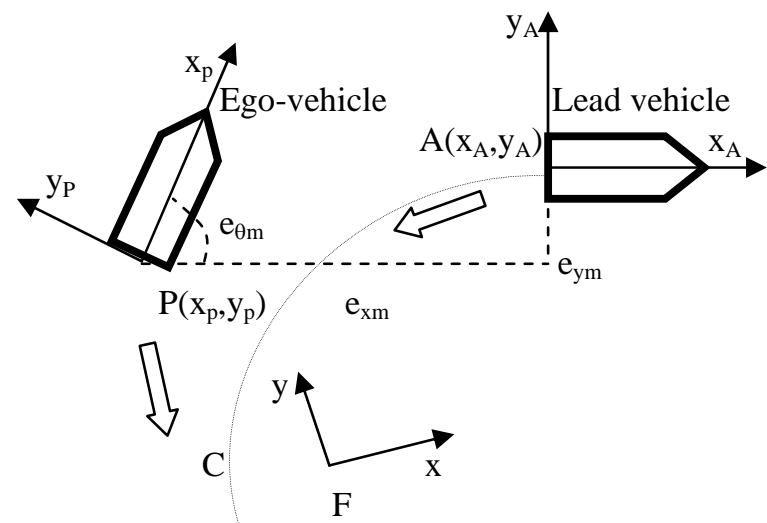

Fig. 1 A plan view of a two-vehicle convoy

We suppose that there is no skidding between the wheels and the ground. Let points $A$ and $P$ located in the center of the rear wheel vehicle axles be the guide points of the leader and the ego-vehicle, respectively. The vehicle postures with respect to an inertial frame Fxy are denoted by $q_{A}=\left[x_{A}, y_{A}, \theta_{R}\right]^{\mathrm{T}} \in \mathfrak{R}^{3}$ and $q_{P}=\left[x_{P}\right.$, $\left.y_{P}, \quad \theta\right]^{\mathrm{T}} \in \mathfrak{R}^{3}$, respectively. The coordinates and orientation of the ego-vehicle in the leader coordinates frame are (Petrov and Shivarov, 1994)

$$
\left[\begin{array}{l}
e_{x m} \\
e_{y m} \\
e_{\theta m}
\end{array}\right]=\left[\begin{array}{ccc}
\cos \theta_{R} & \sin \theta_{R} & 0 \\
-\sin \theta_{R} & \cos \theta_{R} & 0 \\
0 & 0 & 1
\end{array}\right]\left[\begin{array}{c}
x_{P}-x_{A} \\
y_{P}-y_{A} \\
\theta-\theta_{R}
\end{array}\right]
$$

Remark 1. It should be noted that the vehicle-tovehicle distance and relative orientation are measured using distance sensors like a radar that monitor the rear end of the first vehicle, but normally these sensors are installed on the front of the second vehicle. Since we know the position and the orientation of the radars relative to the robot vehicle frame, using geometric relations and coordinate transformations, we can calculate the position and orientation of its frame $P x_{P} y_{P}$ attached to the center $P$ of the rear vehicle axle in the leader vehicle coordinate frame $A x_{A} y_{A}$ placed at the center of the rear vehicle axle.

Differentiating (1) with respect to time and taking into account the nonholonomic constraints, after simple calculations, we obtain

$$
\begin{aligned}
& \dot{e}_{x m}=-v_{A x}+v_{P x} \cos e_{\theta m}+\omega_{R} e_{y m} \\
& \dot{e}_{y m}=v_{P x} \sin e_{\theta m}-\omega_{R} e_{x m} \\
& \dot{e}_{\theta m}=-\omega_{R}+\omega
\end{aligned}
$$


where $\left(v_{A x}, \omega_{R}\right)$ and $\left(v_{P x}, \omega\right)$ are the linear and angular velocities of the first and the second vehicle, respectively. We assume that the lead vehicle moves backward, i.e., $v_{A x}<0$. According to the nonholonomic constraints, the linear velocities $v_{A x}$ and $v_{P x}$ are directed along the longitudinal vehicle axes. The ego-vehicle linear velocity $v_{P x}$ and the front wheel steering angle $\alpha$, are the control inputs of the system, where $\alpha$ is expressed by means of the vehicle angular velocity $\omega$, i.e., $\alpha=\operatorname{atan}\left(l \omega / v_{P x}\right)$, and $l$ is the longitudinal vehicle base.

We assume that we are able to measure the position coordinate $\left(e_{x m}, e_{y \mathrm{~m}}\right)$ and the orientation $e_{\theta m}$ of $P x_{P} y_{P}$ with respect to $A x_{A} y_{A}$. So, the available system output is

$$
y=\left[e_{x m}, e_{y \mathrm{~m}}, e_{\theta m}\right]^{\mathrm{T}} .
$$

In (2), the linear and angular velocities of the lead vehicle $\left(v_{A x}, \omega_{R}\right)$ are unknown constant parameters.

\subsection{Control Objective}

Assuming constant velocity maneuvers for backward driving of the lead vehicle, in this paper, we are interested in a tracking scenario where the twovehicle convoy will travel concentric arcs of same radii with prescribed inter-vehicle spacing depending on linear and angular velocities of the leader. We assume that the linear and angular velocities of the lead vehicle are unknown constant parameters and are not available for feedback control.

First, we assign the desired vehicle-to-vehicle distance $h$, ( $h=c t e$ ), in the case of the straight line maneuver of the leader. Denoting by $\left(v_{A x}, \omega_{R}\right)$ the velocities of the lead vehicle, the desired inter-vehicle spacing during circular maneuvers of constant radius is given by the following expression $s_{d}=h_{v}(1+\cos \varphi)$, where $h_{v}=(l+h) / 2$ and $\varphi=\varphi\left(v_{A x}, \omega_{R}\right)=c t e$ is the relative orientation of the vehicles at steady state. Since the leader velocities are unknown, in this paper, we use their estimates $\left(\hat{v}_{A x}, \hat{\omega}_{R}\right)$ obtained from the dynamic part of the proposed controller to form the reference output for system (2) as

$$
y_{r}=\left[-h_{v}(1+\cos \hat{\varphi}),-h_{v} \sin (\hat{\varphi}), 0\right]^{\mathrm{T}} .
$$

where $\hat{\varphi}=\hat{\varphi}\left(\hat{v}_{A x}, \hat{\omega}_{R}\right)$.

If the velocities of the lead vehicle $\left(v_{A x}, \omega_{R}\right)$ were known, we would be able to determine a reference output for the system (2) of the form $y_{r v}=$ $\left[-h_{v}(1+\cos \varphi),-h_{v} \sin (\varphi), 0\right]^{\mathrm{T}}$ where $\varphi=\varphi\left(v_{A x}, \omega_{R}\right)=c t \epsilon$. Alternatively, this is equivalent to determine the position of a coordinate frame $R_{v} x_{v} y_{v}$ with respect to the leader frame, which satisfies the requirement of traveling circular arc of same radii with prescribed inter-vehicle spacing. In this case, the coordinates and the orientation of this frame with respect to the leader frame are

$$
\left(e_{x v}, \mathrm{e}_{\mathrm{yv}}, e_{\theta v}\right)=\left[-h_{v}(1+\cos \varphi),-h_{v} \sin (\varphi), 0\right]^{\mathrm{T}}
$$

and the relative tracking error dynamics can be developed in terms of $R_{v} x_{v} y_{v}$. It should be noted that reference output (4) could be interpreted as estimates of the coordinates and orientation of $R_{v} x_{v} y_{v}$ with respect to the leader frame which are unknown a priori.

In this paper, we consider the system (2) with the output (3). The control objective is to asymptotically track a reference output (4) with the output (3) of the system.

\section{ADAPTIVE CONTROL DESIGN}

In this paper we consider the problem of controlling the motion of the ego-vehicle during constant velocity maneuvers for backward driving of the lead vehicle. We will treat the problem as an output tracking problem in the presence of unknown constant parameters of the controlled plant.

Using (2) and (3), the tracking error $e=y-y_{r},(e=$ $\left.\left[e_{x}, e_{y}, e_{\theta}\right] \in \mathfrak{R}^{3}\right)$, is given by

$$
\begin{aligned}
& e_{x}=e_{x m}+h_{v}(1+\cos \hat{\varphi}) \\
& e_{y}=e_{y m}+h_{v} \sin \hat{\varphi} \\
& e_{\theta}=e_{\theta m}
\end{aligned}
$$

Differentiating (6) with respect to time and using (2), we obtain the tracking error dynamics in the form

$$
\begin{aligned}
& \dot{e}_{x}=-v_{A x}+v_{P x} \cos e_{\theta}+\omega_{R}\left(e_{y}-h_{v} \sin \hat{\varphi}\right)-h_{v} \dot{\hat{\varphi}} \sin \hat{\varphi} \\
& \dot{e}_{y}=v_{P x} \sin e_{\theta}-\omega_{R}\left[e_{x}-h_{v}(1+\cos \hat{\varphi})\right]+h_{v} \dot{\hat{\varphi}} \cos \hat{\varphi} \\
& \dot{e}_{\theta}=-\omega_{R}+\omega
\end{aligned}
$$

where the linear and angular velocities of the lead vehicle, $\left(v_{A x}, \omega_{R}\right)$, are unknown constant parameters. Note that, because the reference output $y_{r}(t)$ is not constant, its derivative appears in the tracking error dynamics. We suppose that $\left|\mathrm{e}_{\theta}\right|<\pi / 2$ which is the case when the two vehicles do not travel in opposite directions.

To facilitate the subsequent control design and corresponding stability proof, we define the following state transformation

and input transformations

$$
\mu=\tan e_{\theta}
$$

$$
\begin{gathered}
u_{1}=v_{P X} \cos _{\theta} \\
u_{2}=\frac{\omega-\hat{\omega}_{R}}{\cos ^{2}(a \tan \mu)}
\end{gathered}
$$


Using the state transformation as defined in (8) and the input transformation (9), the system (7) can be written in the form

$$
\begin{aligned}
& \dot{e}_{x}=-v_{A x}+u_{1}+\omega_{R}\left(e_{y}-h_{v} \sin \hat{\varphi}\right)-h_{v} \dot{\hat{\varphi}} \sin \hat{\varphi} \\
& \dot{e}_{y}=-\omega_{R}\left[e_{x}-h_{v}(1+\cos \hat{\varphi})\right]+h_{v} \dot{\hat{\varphi}} \cos \hat{\varphi}+u_{1} \mu \\
& \dot{\mu}=u_{2}+\frac{\tilde{\omega}_{R}}{\cos ^{2}(a \tan \mu)}
\end{aligned}
$$

where $\tilde{v}_{A x}$ and $\widetilde{\omega}_{R}$ are the parameter errors

$$
\begin{aligned}
& \tilde{v}_{A x}=\hat{v}_{A x}-v_{A x} \\
& \tilde{\omega}_{R}=\hat{\omega}_{R}-\omega_{R}
\end{aligned}
$$

In this paper, we propose an adaptive control law designed on a reduced order model combined with high-gain design method (Kristic et al., 1995; Sepulchre et al. 1997) which achieves asymptotic stabilization of the closed-loop system in error coordinates. Since the leader velocities are not available for feedback control design, the control velocities of the ego-vehicle are computed using the leader velocity estimates obtained from the dynamic part of the proposed controller.

The adaptive control design is based on a reducedorder system composed of the first two equations of (11). The idea is to use $u_{1}$ and $\mu$ as the first pair of controls. Consider the subsystem (11a) where $u_{A x}<0$, and assume that the leader velocities $v_{A x}=$ cte and $\omega_{R}$ = cte, are unknown constant parameters. The control problem consists in finding an adaptive feedback control law for the system (10a) with inputs $\left(u_{1}, \mu\right)$ such that $\lim _{t \rightarrow \infty}\left(y_{i}(t)-y_{r i}(t)\right)=0, \quad i=1,2$.

We propose the following feedback control

$u_{1}=\hat{v}_{A x}-\hat{\omega}_{R}\left(e_{y}-h_{v} \sin \hat{\varphi}\right)+h_{v} \dot{\hat{\varphi}} \sin \hat{\varphi}-k_{1} e_{x}$

$u=\frac{1}{u_{1}}\left\{\hat{\omega}_{R}\left[e_{x}-h_{v}(1+\cos \hat{\varphi})\right]-h_{v} \dot{\hat{\varphi}} \cos \hat{\varphi}-c_{1} e_{y}\right\}$

where $u:=\mu_{d}=\mu_{d}\left(e_{x}, e_{y}, \hat{v}_{A x}, \hat{\omega}_{R}\right) ; k_{1}$ and $c_{1}$ are positive gains.

We consider the following Lyapunov function candidate

$$
V=\frac{1}{2} e_{x}^{2}+\frac{1}{2} e_{y}^{2}+\frac{1}{2 \gamma_{e x}} \tilde{v}_{R x}^{2}+\frac{1}{2 \gamma_{e y}} \tilde{\omega}_{R}^{2}
$$

where $\gamma_{e x}>0$ and $\gamma_{e y}>0$ are the adaptation gains.

Using (13) and (14), the derivative of $V$ is

$$
\begin{aligned}
\dot{V}= & -k_{1} e_{x}^{2}-c_{1} e_{y}^{2}+\tilde{v}_{A x}\left(e_{x}+\frac{1}{\gamma_{e x}} \dot{\hat{v}}_{A x}\right) \\
& +\tilde{\omega}_{R}\left[h_{v} e_{x} \sin \hat{\varphi}-h_{v} e_{y}(1+\cos \hat{\varphi})+\frac{1}{\gamma_{e y}} \dot{\hat{\omega}}_{R}\right]
\end{aligned}
$$

where all the terms containing $\widetilde{v}_{A x}$, as well as $\tilde{\omega}_{R}$, have been grouped together. To eliminate them, the update law is chosen as

$$
\begin{aligned}
& \dot{\hat{v}}_{A x}=-\gamma_{e x} e_{x} \\
& \dot{\hat{\omega}}_{R}=\gamma_{e y}\left[h_{v} e_{x} \sin \hat{\varphi}-h_{v} e_{y}(1+\cos \hat{\varphi})\right]
\end{aligned}
$$

and we obtain for the derivative of $V$

$$
\dot{V}=-k_{1} e_{x}^{2}-c_{1} e_{y}^{2} \leq 0
$$

In (13) and (14), for $\dot{\hat{\varphi}}\left(\dot{\hat{v}}_{A x}, \dot{\hat{\omega}}_{R}\right)$, we use the analytical expressions of the update law (17). The resulting closed-loop adaptive system becomes

$$
\begin{aligned}
& \dot{\hat{v}}_{A x}=-\gamma_{e x} e_{x} \\
& \dot{\hat{\omega}}_{R}=\gamma_{e y}\left[h_{v} e_{x} \sin \hat{\varphi}-h_{v} e_{y}(1+\cos \hat{\varphi})\right] \\
& \dot{e}_{x}=-k_{1} e_{x}+\widetilde{v}_{A x}-\widetilde{\omega}_{R}\left(e_{y}-h_{v} \sin \hat{\varphi}\right) \\
& \dot{e}_{y}=-c_{1} e_{y}+\widetilde{\omega}_{R}\left[e_{x}-h_{v}(1+\cos \hat{\varphi})\right]
\end{aligned}
$$

where $\dot{\hat{v}}_{A x}=\dot{\widetilde{v}}_{A x}$ and $\dot{\hat{\omega}}_{R}=\dot{\tilde{\omega}}_{R}$.

Proposition 1. Assume that lead vehicle's linear and angular velocities $\left(v_{A x}, \omega_{R}\right)$ are unknown constant parameters, ant that $v_{A x} \leq V_{\text {cte }}<0$. If the control law given by (13)-(14) is applied to (11a) where the leader velocity estimates $\left(\hat{v}_{A x}, \hat{\omega}_{R}\right)$ are obtained from the parameter update law (17), the origin $x=\left[e_{x}, e_{y}, \tilde{v}_{A x} \tilde{\omega}_{R}\right]^{T}=$ 0 of the closed-loop system (19) in error coordinates is asymptotically stable.

Proof. Based on the LaSalle's invariance principle, the stability properties of (19) follow from (15) and (18). Let $\mathrm{D}=\left\{x=\left[e_{x}, e_{y}, \tilde{v}_{A x}, \tilde{\omega}_{R}\right]^{T} \in \mathfrak{R}^{4} \mid\right.$ $\left.\left|\hat{\varphi}\left(\hat{v}_{A x}, \hat{\omega}_{R}\right)\right|<\pi\right\}$. The system has an equilibrium point at the origin. The function $V(t): D \rightarrow \Re$ is continuously differentiable and positive definite. From (18), it follows that (15) is nonincreasing, $(V(t) \leq V(0))$, and this in turn implies that $e_{x}(t), e_{y}(t), \widetilde{v}_{A x}(t)$ and $\tilde{\omega}_{R}(t)$ are bounded and converge to the largest invariant set $M$ of (19) contained in the set $E=\{\mathrm{x} \in \mathrm{D}$ $\left.\mid \dot{V}=-k_{1} e_{x}^{2}-c_{1} e_{y}^{2}=0\right\}$. Suppose $x(t)$ is a trajectory that belongs identically to $E$. But, $\left(e_{x}(t) \equiv 0, e_{y}(t) \equiv 0\right) \Rightarrow$ $\left(\dot{e}_{x}(t) \equiv 0, \dot{e}_{y}(t)=0\right)$ which, in turn implies (from the first and the second equations of (19)) that $\widetilde{v}_{A x}(t) \equiv 0, \tilde{\omega}_{R}(t) \equiv 0$. Therefore, from (Khalil, 1996; Corollary 3.1, p.116), the only solution that can stay identically in $E$ is the trivial solution $x(t)=0$. Thus, the origin is asymptotically stable, i.e.

$$
\lim _{t \rightarrow \infty} x=\left[e_{x}(t), e_{y}(t), \tilde{v}_{A x}(t), \tilde{\omega}_{R}(t)\right]^{T}=0
$$


In addition, based on the linearization of (19) at the origin, it can be shown that $x(t)=0$ is exponentially stable equilibrium point for the corresponding linear system. Using (Khalil, 1996; Theorem 3.11, p.147) we conclude that the proposed adaptive control achieves local exponential stability of $x=0$ for the nonlinear system (19).

To stabilize the complete system given by (11b)(19), we use high-gain design. Denoting by $x=\left[e_{x}, e_{y}, \tilde{v}_{A x}, \tilde{\omega}_{R}\right]^{T}$ and using (12), the system (19) can be represented in the form

$$
\dot{x}=f(x)+g(x) u
$$

where $\hat{v}_{A x}=\tilde{v}_{A x}+v_{A x}, \quad \hat{\omega}_{R}=\widetilde{\omega}_{R}+\omega_{R}, \quad \hat{\varphi}=\hat{\varphi}\left(\hat{v}_{A x}, \hat{\omega}_{R}\right)$

$$
\begin{aligned}
& f(x)=\left[\begin{array}{c}
-\gamma_{e x} e_{x} \\
\gamma_{e y}\left[h_{v} e_{x} \sin \hat{\varphi}-h_{v} e_{y}(1+\cos \hat{\varphi})\right] \\
-k_{1} e_{x}+\tilde{v}_{A x}-\widetilde{\omega}_{R}\left(e_{y}-h_{v} \sin \hat{\varphi}\right) \\
-\omega_{R}\left[e_{x}-h_{v}(1+\cos \hat{\varphi})\right]+h_{v} \hat{\varphi}_{\text {dot }} \cos \hat{\varphi}
\end{array}\right] \\
& g(x)=\left[\begin{array}{c}
0 \\
0 \\
0 \\
\hat{v}_{A x}-\hat{\omega}_{R}\left(e_{y}-h_{v} \sin \hat{\varphi}\right)+h_{v} \hat{\varphi}_{d o t} \sin \hat{\varphi}-k_{1} e
\end{array}\right]
\end{aligned}
$$

In (21), $u:=\mu_{d}(x)$ given by (14), achieves local exponential stability of $x(t)=0$.

The last equation of (11) can be written in the form

$$
\dot{\mu}=a(x, \mu)+u_{2}
$$

where

$$
a(x, \mu)=\frac{\tilde{\omega}_{R}}{\cos ^{2}(a \tan \mu)}
$$

Since $u_{1}$ is an actual control variable, we can use (13) as control law for the system (11a). However, this is not the case with $\mu$, (eq. (14)), because it is a state variable. To approximately implement (14), we use high-gain controller in the form

$$
u_{2}=-K\left[\mu-\mu_{d}(x)\right]
$$

where $\mu_{d}(x)$ is given by (14).

To establish asymptotic stability for the augmented system, we consider the system composed of (21) - (22) rewritten in the form

$$
\begin{aligned}
& \dot{x}=f(x)+g(x) \mu \\
& \dot{\mu}=a(x, \mu)+u_{2}
\end{aligned}
$$

We introduce a new variable

$$
\zeta:=\mu-\mu_{d}(x)
$$

Based on Proposition 6.5 (Sepulchre, 1997; p.244) , we conclude that the control (23) achieves semi-global stabilization of $(x, \zeta)=(0,0)$ for the system $(24)$ in the new coordinates (25). Hence

$$
[x, \zeta]^{T}=\left[e_{x}(t), e_{y}(t), \tilde{v}_{A x}(t), \tilde{\omega}_{R}(t), \zeta(t)\right]^{T} \rightarrow 0 \text { as } t \rightarrow \infty
$$

From (26), it follows for the relative orientation of the vehicles at steady state is

$$
\hat{\varphi}_{s s}\left(\hat{v}_{A x}, \hat{\omega}_{R}\right)=\varphi\left(v_{A x}, \omega_{R}\right)=\text { cte. }
$$

The inter-vehicle spacing during circular maneuvers of constant radius is given by the expression $s_{d}=h_{v}\left(1+\cos \hat{\varphi}_{s s}\right)$. Finally, from (4), (5), (6), (26) and (27), it also follows that the coordinates of the egovehicle $\left(e_{x m}, e_{y m}\right)=\left(e_{x}-h_{v}(1+\cos \hat{\varphi}), e_{y}-h_{v} \sin \hat{\varphi}\right) \quad$ with respect to the leader frame $A x_{A} y_{A}$ converge to the coordinates $\left(-h_{v}(1+\cos \varphi),-h_{v} \sin \varphi\right)$ of point $R_{v}$ (the frame $\left.R_{v} x_{v} y_{v}\right)$ in the leader frame.

\section{SIMULATION RESULTS}

To illustrate the effectiveness of the proposed controller, several simulations are carried out in order to evaluate the vehicle behavior and tracking accuracy. In the simulation, a planar bicycle 2DOFs vehicle model is used. The parameters of the system are assumed to be

- longitudinal vehicle base - $2 m$;

- desired inter-vehicle separation in the case of straight line motion of the lead vehicle - $1.5 \mathrm{~m}$;

The leader path consists of three constant velocity maneuvers of fixed duration (25 seconds each): turning both to the left and to the right followed by a straight line motion. The corresponding leader velocities are as follows:

\begin{tabular}{|l|c|c|}
\hline & Linear velocity & Angular velocity \\
\hline Maneuver 1 & $-1 \mathrm{~m} / \mathrm{s}$ & $0.1 \mathrm{rad} / \mathrm{s}$ \\
\hline Maneuver 2 & $-1 \mathrm{~m} / \mathrm{s}$ & $-0.1 \mathrm{rad} / \mathrm{s}$ \\
\hline Maneuver 3 & $-1.5 \mathrm{~m} / \mathrm{s}$ & $0 \mathrm{rad} / \mathrm{s}$ \\
\hline
\end{tabular}

The initial estimates for the leader linear and angular velocities are $-0.6 \mathrm{~m} / \mathrm{s}$ and $0 \mathrm{rad} / \mathrm{s}$, respectively, and are different from the true values. The initial position and orientation of the ego-vehicle in the leader coordinate frame are $(-3 m, 0.25 m)$ and 0.1rad, respectively.

Simulation results are shown in Figures 2, 3 and 4. From Figure 2, we can see the planar paths drown by the vehicle guide points. As shown in Figure 3, the estimates of the leader velocities tend asymptotically to their actual values. From Figure 4a, we can see the evolution of the inter-vehicle spacing. At steady state, the spacing is $1.515 \mathrm{~m}$ for circular motion of radius $10 \mathrm{~m}$, and $1.5 \mathrm{~m}$ for rectilinear motion of the leader. As shown in Figure 4b, the tracking errors tend asymptotically to zero; the robot vehicle orientation with respect to the leader also tends asymptotically to a desired value. This 
value depends on the curvature radius of the path traveled by the leader. The results of the simulation verify the validity of the proposed controller.

\section{CONCLUSION}

In this paper, an adaptive tracking controller for a two-vehicle convoy where the lead vehicle is driven in reverse has been presented. We assume that the lead vehicle linear and angular velocities are unknown constant parameters. We discuss autonomous vehicle control without any information obtained from road infrastructure or communicated from the lead vehicle. The only information the robot vehicle can use for feedback control is the relative position and orientation with respect to the lead vehicle obtained from onboard sensing. The control velocities of the ego-vehicle are computed using the leader velocity estimates. For constant velocity maneuvers of the leader, at steady state, the two-vehicle convoy will travel concentric arcs of same radii with prescribed inter-vehicle spacing. The results of the simulation verify the validity of the proposed controller. Future research will address the problem of controlling the backward driving of the vehicle convoy in the case of unknown time-varying velocities of the lead vehicle.

\section{REFERENCES}

Cheok, K., G.Smidt, KKobayashi, J.Overholt, P. Lescoe (1997).“A fuzzi logic intelligent control system paradigm for an in-line-ofsight leader-following HMMWV", Journal of Robotic Systems, 14(6), 1997, pp. 407-420.

Brumitt, B., M.Hebert (1998). "Experiments in autonomous Driving with concurrent goals and multiple vehicles", Proc. IEEE Int. Conf. Rob. Automation, pp.1895-1902.

Hogg, R., A. Rankin, M. McHenry, D. Helmick, C. Bergh, S. Roumeliotis, L. Matthies (2001). "Sensors and actuators for small robot leader/follower behavior", $15^{\text {th }}$ AeroSense Symposium, April.

Fritz, H. (1999). "Longitudinal and lateral control of heavy duty trucks for automated vehicle following in mixed traffic: experimental results from the CHAUFFEUR project”, Proc Int. Conf. Contr. Applications, pp.1348-1352.

Parent, M., P. Daviet (1996). ” Automated urban vehicles: towards a dual mode PRT (Personal Rapid Transit)", Proc. IEEE Conf. Rob. Automation, pp.3129-3133.

Lu, G. ,J. Huang, M.Tomizuka, (2004). "Vehicle lateral control under fault in front and/or rear sensors:final report" , Reserch Report, UCB-ITS-PRR-2004-36.

White, R., M. Tomizuka (2001). "Autonomous following lateral control of heavy vehicles using laser scanning radar", Proc. American Control Conference, pp. 2333-2337.

Canudas-de-Wit, C., A. NDoudi-Likoho (2000). Nonlinear control for a convoy-like vehicle, Automatica 36, pp. 457-462.

Artus, G., P. Morin, C. Samson (2003). "Tracking of an omnidirectional target with a nonholonomic mobile robot”, Proc. Int. Conf. on Advanced Robotics, pp. 1468-1473.

Kristic, M., I. Kanellakopoulos, P. Kokotovic (1995). Nonlinear and Adaptive Control Design, Wiley, New York.

Sepulchre, R., M. Jankovic, P. Kokotovic, (1997). Constructive Nonlinear Control, Springer-Verlag London Ltd .

Petrov, P., N. Shivarov (1994), "Path control of an autonomous wheeled mobile robot”, Problems of Eng. , Cyb. and Robotics, vol. 41, pp.124-128.

Khalil, H. (1996). Nonlinear Systems, Prentice Hall.

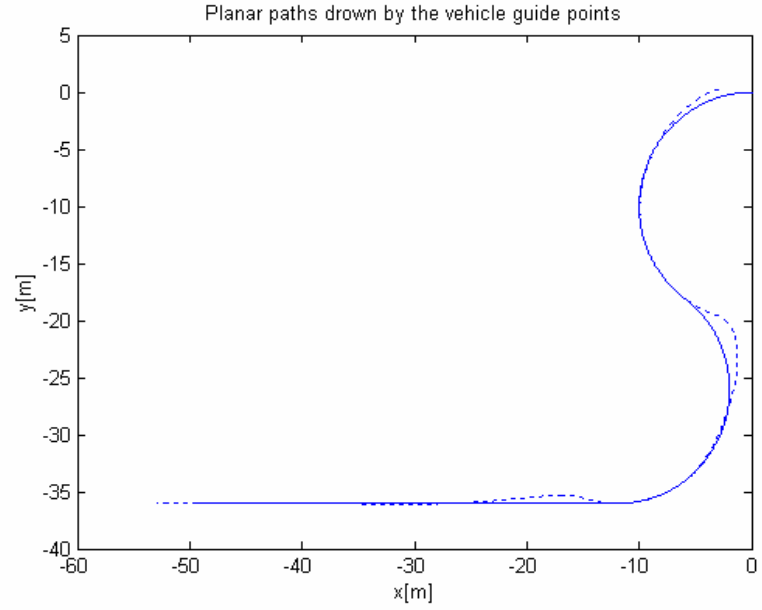

Fig. 2 A planar path drown by the vehicle guide points; Continuous line (leader), dashed line(robot vehicle)
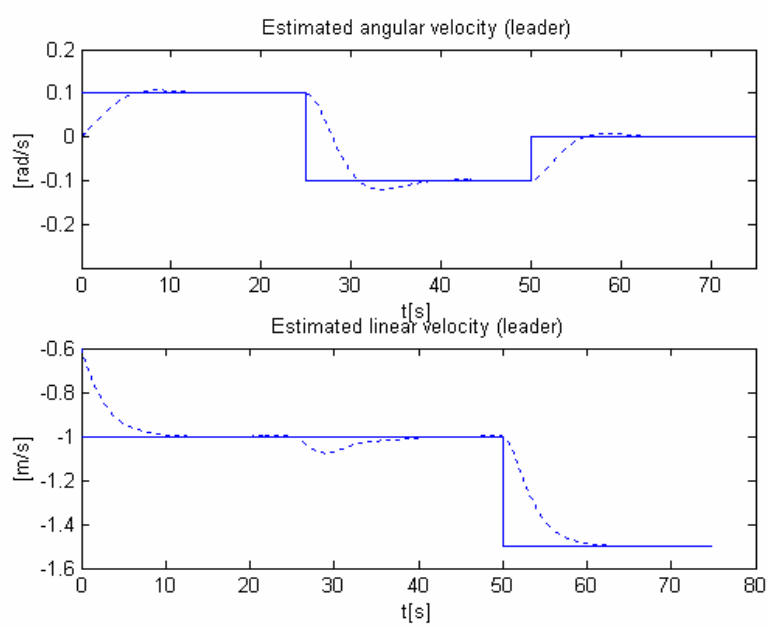

Fig. 3 Leader actual velocities (continuous line) and velocities estimates (dashed line)
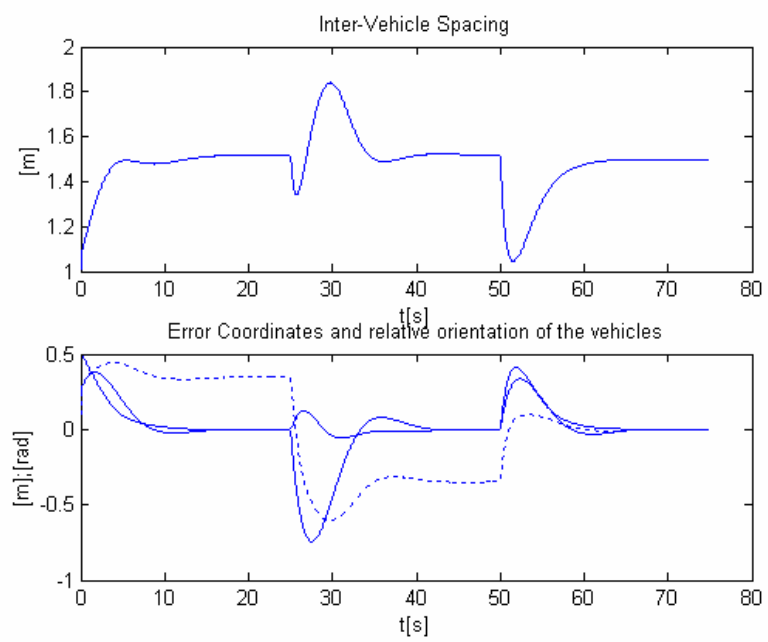

Fig. 4 Evolution of the inter-vehicle spacing (Fig. 3a); Evolution of the tracking errors (continuous lines) and the orientation of the robot vehicle with respect to the leader (dashed line), (Fig. 3b) 